\title{
Le fibrinogène, facteur multifonctionnel de l'hémostase
}

Le fibrinogène joue un rôle important à différents niveaux de la coagulation sanguine; il est non seulement un facteur essentiel à la formation du caillot, mais module aussi l'activité des plaquettes sanguines et des cellules endothéliales. Des informations récentes ont été acquises sur la structure et l'expression des gènes du fibrinogène.

\section{Gérard Marguerie}

Directeur de l'Unité Inserm 217 et du laboratoire d'hématologie, Centre d'études nucléaires de Grenoble.

\section{ADRESSE}

G. Marguerie : Département de recherche fondamentale, laboratoire d'hématologie, unité Inserm 21 7, C.E.N.G.-85 X, 3804I Grenoble Cedex.

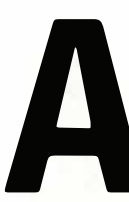

$\mathrm{u}$ cours des trente dernières années, de considérables travaux ont été consacrés au fibrinogène, protéine majoritaire du plasma. Le comportement de la protéine en solution et la séquence des chaînes polypeptidiques qui la constituent ont été déterminés. Les réactions du fibrinogène avec la thrombine, la plasmine et le facteur XIIIa ont été amplement étudiées. Le rôle de certains dérivés physiologiques du fibrinogène ou de la fibrine dans plusieurs syndromes hémorragiques ou thrombotiques a été analysé. L'importance de ce facteur dans l'activité de la cellule endothéliale et de la plaquette a été mise en évidence. Enfin les développements de la génétique moléculaire ont permis d'accéder aux gènes de cette protéine et l'étude de leur structure et de leur expression a commencé. Loin d'altérer l'intérêt que l'on pouvait porter à ce facteur de la coagulation, cette masse d'informations a révélé que cette protéine remplit plusieurs fonctions dans l'hémostase et représente un modèle unique pour l'étude de l'expression génétique d'une protéine multicaténaire.

La molécule est une glycoprotéine de poids moléculaire 340000 , constituée de deux sous-unités, chacune d'elles comportant trois chaînes polypeptidiques dont les séquences sont connues $[\mathrm{I}, 2]: \mathrm{A} \alpha$ (PM 66.000, 610 aa), B $\beta$ (PM $54.000,46 \mathrm{I}$ aa) et $\gamma\left(\mathrm{PM}_{4} 8.000\right.$, 4 I I aa) (figure I).

Un polymorphisme moléculaire concernant les trois chaînes peptidiques de la protéine a été mis en évidence. L'existence de plusieurs chaînes $A \alpha$ circulantes n'a pas, à ce jour, d'explication satisfaisante. Elles seraient le résultat d'une dégradation protéolytique partielle de la chaîne native. Dans le cas de la chaine $B \beta$ il pourrait s'agir d'un degré de glycosylation variable. L'origine du polymorphisme de la chaîne $\gamma$ est par contre mieux 


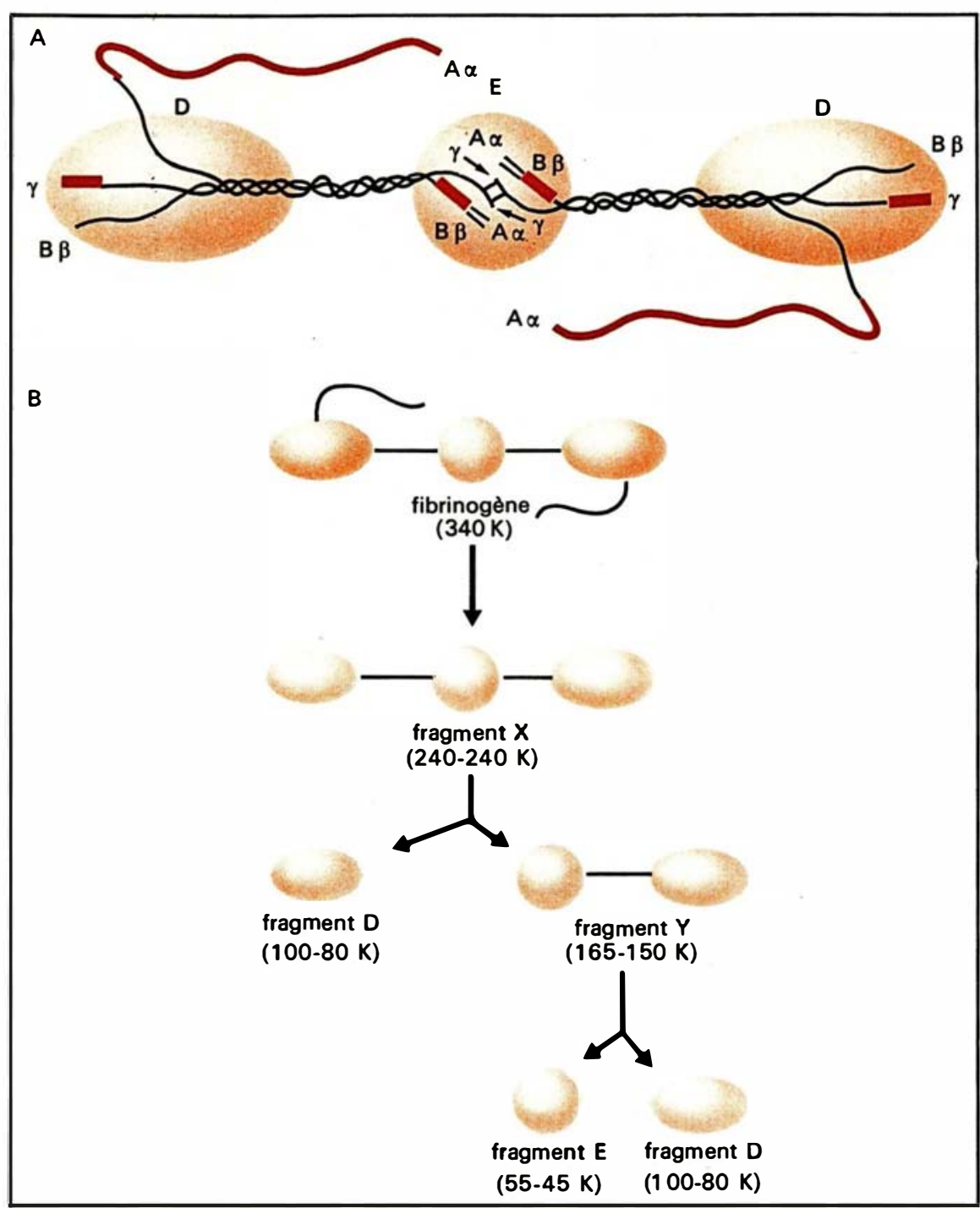

Figure 1. La molécule de fibrinogène et sa dégradation par le plasmine. A) Les trois paires de chaînes $A \alpha, B \beta$ et $\gamma$ sont organisées en un domaine central $E$ qui contient toutes les extrémités $N$-terminales et deux domaines périphériques $D$ qui contiennent les extrémités C-terminales. B) L'action de la plasmine sur la protéine est asymétrique. Elle produit des fragments de dégradation précoces $X$ et $Y$ et des fragments tardifs $D$ et $E$.

connue. Il existe deux variants $\gamma$ et $\gamma^{\prime}$ qui different entre eux par leur extrémité C-terminale. Chez l'homme, les quatre derniers acides aminés de la chaîne $\gamma$ sont remplacés par vingt autres dans $\gamma^{\prime}[3]$. La synthèse de cette chaîne résulte d'un phénomène d'épissage alternatif. Dans $\gamma^{\prime}$, le dernier exon est remplacé par une partie du dernier intron non excisé. Les 20 premiers codons sont traduits en protéine du fibrinogène plasmatique d'origine hépatique, mais d'une expression des gènes de cette protéine dans le mégacaryocyte [5]. Fibrinogènes plasmatique et plaquettaire ont des caractéristiques structurales identiques. Mais des différences ont été observées : réactivités immunochimiques différentes et absence du variant $\gamma^{\prime}$ dans la plaquette. De plus, certaines anomalies congénitales décrites pour le fibrinogène plasmatique n'ont pas été retrouvées au niveau de la plaquette [6]. Ces observations suggèrent que l'expression génétique et/ou les maturations post-traductionnelles de la protéine dans les tissus hépatiques et médullaires sont différentes.

\section{Synthèse et régulation}

Le fibrinogène plasmatique est synthétisé et secrété par les cellules du parenchyme hépatique. Son taux varie de 2 à $4 \mathrm{~g} / \mathrm{l}$ et sa demi-vie est de 3 à 5 jours. Les étapes qui conduisent à la synthèse et à l'assemblage de la molécule ont été partiellement étudiées. Les trois chaînes du fibrinogène sont synthétisées avec des cinétiques comparables et passent directement dans la lumière du réticulum endoplasmique par l'intermédiaire de leur peptide signal. La glycosylation de la chaîne $\gamma$ est assurée au cours de cette traversée. Celle de la chaine $\mathrm{B} \beta$ est plus tardive et n'est réalisée qu'en fin de traduction. La molécule est assemblée dans le réticulum endoplasmique avant d'être exportée vers le plasma [7].

$\mathrm{La}$ synthèse de la protéine augmente à la suite d'un stress, au cours de la grossesse, lors d'une blessure ou après injection d'endotoxine, de prostaglandine ou d'hormone de croissance. Cette production accrue de fibrinogène est due à une autorégulation dont le mécanisme est à peine connu [8]. Au cours de la phase aiguë de la réponse inflammatoire il y a transformation du fibrinogène en fibrine et activation du système fibrinolytique. Les fragments $D$ et $E$ de fibrinolyse ou de fibrinogénolyse stimulent certains récepteurs non identifiés sur la membrane de la cellule de Kupffer ou des leucocytes (figure 2, p. suivante). Cette stimulation induit la 


\section{REFEERENCES}

r. Doolittle RF, Goldman DM, Doolittle LR. Designation of sequences involved in the coiledcoil interdomanial connections in fibrinogen. $\mathcal{F}$ Mol Biol 1978; 120, 3 11-25.

2. Henshen A, Lottspeich F, Kehl M, Southan C. Covalent structure of fibrinogen. $A n n N Y$ Acad Sci 1983; 408: 28-43.

3. Wolfenstein-Todel C, Mossesson MW. Carboxyterminal amino acid sequence of a human fibrinogen chain variant $\left(\gamma^{\prime}\right)$ Biochemistry $198 \mathrm{I}$; 20: 6146-9.

4. Chung DW, Davie EW: $\gamma$ and $\gamma^{\prime}$ chain of human fibrinogen are produced by alternative mRNA processing. Biochemistry 1984; 23: 4232-6.

5. Uzan G, Kerbiriou D, Stankovic Z, Courtois G, Crabree G, Marguerie G. Expression of fibrinogen in megakaryocyte. Thromb Haemost 1985; 54: 278.

6. Jandrot Perrus $M$, Mossesson MW, Denninger MH, Menache D. Studies of platelet fibrinogen from a subject with a congenital plasma fibrinogen abnormality. Blood 1979; 54: 1 109-6.

7. Fuller GM, Nickerson JM, Adams MA. Translational and cotranslational events in fibrinogen synthesis. Ann NY Acad Sci 1983; 408: 440-7.

8. Fuller GM, Ritchie DG. A regulatory pathway for fibrinogen biosynthesis involving an indirect feedback loop. Ann NY Acad Sci 1 982; 389: 308-2.

9. Nickerson JM, Fuller GM. In vitro synthesis of rat fibrinogen. Proc Natl Acad Sci USA 1981; 78: 303-7.

10. Crabtree GR, Kant JA. Molecular cloning of cDNA for the $\alpha, \beta$ and $\gamma$ chains of rat fibrinogen. 7 Biol Chem 1981; 256: 9718-23.

11. Uzan G, Besmond C, Kahn A, Marguerie G. Cell free synthesis of human fibrinogen. Biochem Int 1982; 4: 271-27.

12. Henry I, Uzan G, Weil D, et al. The genes coding for $A \alpha, B \beta$ and $\gamma$ chains of fibrinogen Map to 492. Am 7 Hum Genet 1984; 36: 760-8.

13. Kant JA, Fornace AJ, Saxe D, Simon MI, McBride OW, Crabtree GR. Evolution and organization of the fibrinogen locus on chromosome 4 gene duplication accompanied by transposition and inversion. Proc Natl Acad Sci USA 1985; 82: 2344-8.

14. Courtois G, Uzan G, Assouline Z, Kahn A, Marguerie G. Résultats non publiés.

15. Humphries SE, Iman AMA, Robbins TP, et al. The identification of a DNA polymorphism of the $\alpha$ fibrinogen gene, and the regional assignement of the human fibrinogen genes to 4926-qter.

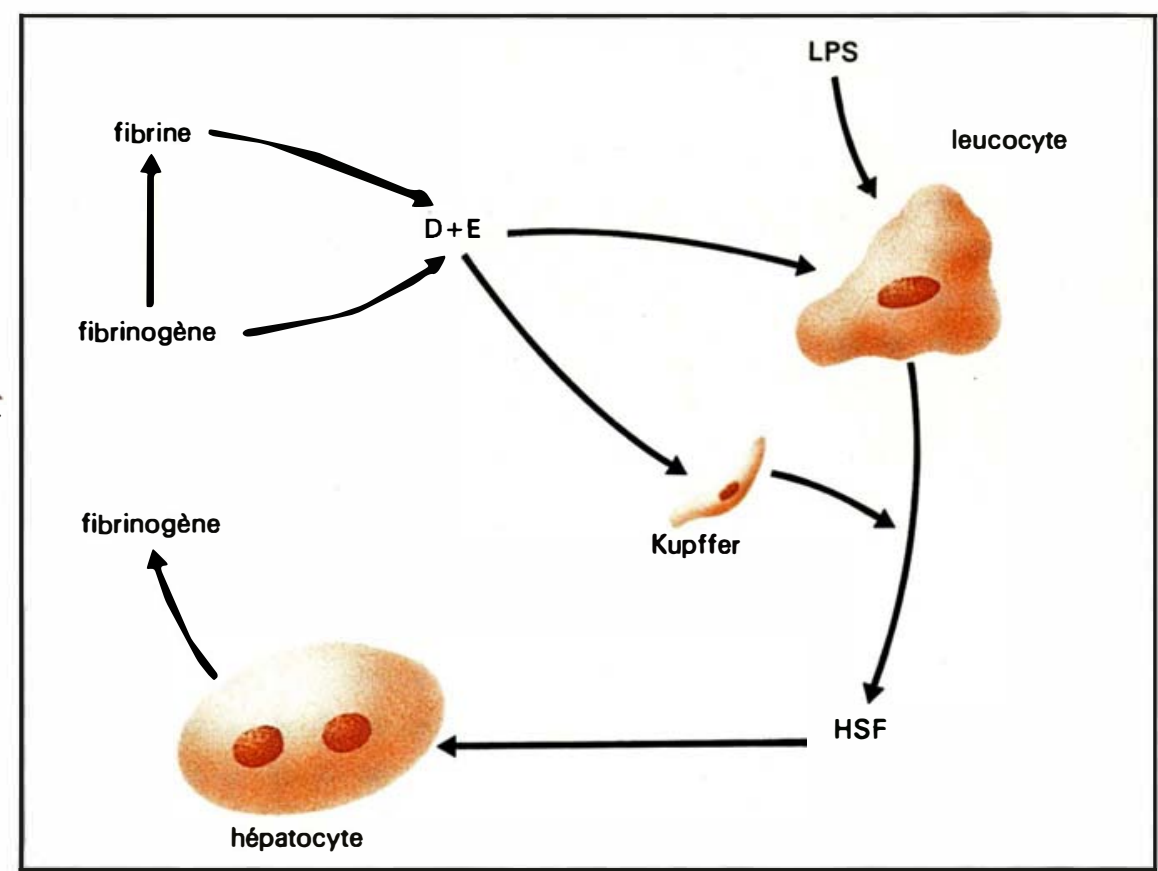

Figure 2. Schéma hypothétique de l'autorégulation de la synthèse du fibrinogène au cours de la phase aiguë de la réponse inflammatoire. HSF= hepatocyte stimulating factor. $L P S=$ lipopolysaccharides.

production d'un facteur de PM 20000 à 40000 , le HSF, ou facteur stimulant de l'hépatocyte, différent des interleukines, qui stimule la synthèse du fibrinogène par l'hépatocyte. Ce mécanisme fait peut-être intervenir les glucocorticoïdes car l'action du HSF est très fortement réduite chez le rat surrénalectomisé. Cette stimulation n'est pas spécifique car d'autres agents, comme les lipopolysaccharides (LPS) peuvent induire la synthèse du HSF et le HSF peut stimuler la synthèse de marqueurs de la réponse inflammatoire autres que le fibrinogène, comme l'haptoglobine. Ce mécanisme n'en demeure pas moins un modèle extrêmement intéressant d'autorégulation.

\section{Structure et organisation des gènes}

Les trois chaînes du fibrinogène sont codées par trois ARNm qui possèdent en $5^{\prime}$ une région codant pour un peptide signal [9-I I]. La protéine est donc le produit de trois gènes différents et l'obtention d'ADN complémentaires spécifiques de chaque chaîne a permis de localiser ces gènes dans la région 4 q2 du chromosome 4 [I2]. Il n'existe qu'une copie des gènes et aucun pseudogène n'a été identifié. Cette situation est rarement observée pour des protéines multicaténaires dont les gènes de structure sont en général mieux répartis dans le génome. Chaque gène couvre entre 8 et ı $\mathrm{kb}$. Leur répartition introns-exons est relativement dense et en relation avec les différents domaines de structure de la protéine. Les gènes sont colinéaires avec une organisation de type $\gamma-\alpha-\beta$ (figure 3). Chez l'homme, les gènes $\gamma$ et $\alpha$ sont distants de ro kb, les gènes $\alpha$ et $\beta$ de I $2 \mathrm{~kb}$. Le gène $\beta$ se trouve orienté dans un sens inverse de celui des deux autres gènes $[13,14]$. Ces gènes sont en cours d'étude dans plusieurs laboratoires dont le nôtre et les informations s'accumulent. Plusieurs particularités ont déjà été identifiées. Un polymorphisme, lié à un site TaqI a 


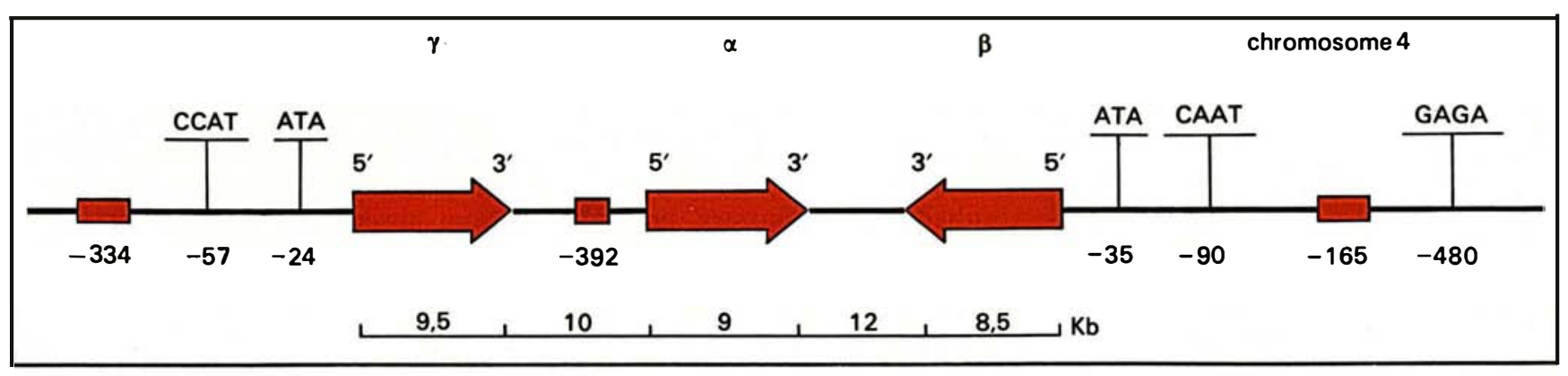

Figure 3. Organisation du locus fibrinogène. Ce schéma résume l'ensemble des informations obtenues à ce jour, chez l'homme et le rat. Les gènes codant pour chaque chaîne peptidique sont organisés selon une orientation $\gamma-\alpha-\beta$ sur une portion réduite du chromosome 4. Des séquences promotrices ont été identifiées pour les gènes $\gamma$ et $\beta$. Des séquences homologues sont également présentées aux positions $-334,-392$ et -165 des gènes $\gamma, \alpha$ et $\beta$ respectivement.

été détecté dans la région $3^{\prime}$ du gène $\alpha$ [i 5]. Il existe une séquence TATA en $5^{\prime}$ des gènes $\beta$ et $\gamma$ à la position attendue, mais rien de tel sur le gène $\alpha$. Des séquences analogues à la séquence CAAT peuvent être localisées aux positions attendues pour les gènes $\beta$ et $\gamma$. Mais aucune séquence de ce genre n'est apparente dans le gène $\alpha$ [I6]. Il serait simple d'imaginer que le gène $\alpha$ est sous le contrổe des séquences promotrices du gène $\gamma$, mais ceci est peu probable. Il existe en effet, dans la partie $5^{\prime}$ du gène $\alpha$ des séquences qui n'ont pas encore été correctement identifiées mais qui possèdent une activité promotrice détectable à l'aide de la méthode de transcription in vitro. Par ailleurs, chez le rat, des séquences homologues entre les gènes $\alpha, \beta$ et $\gamma$ ont été identifiées dans les régions flanquantes $5^{\prime}\left[{ }^{1} 7\right]$. Il est par conséquent tentant de supposer que ces séquences homologues, qui auraient été conservées, pourraient avoir une activité régulatrice pour chacun des gènes. Mais ceci reste à démontrer. Enfin une séquence analogue aux séquences décrites pour les gènes sensibles aux glucocorticoïdes a été découverte dans la région $5^{\prime}$ du gène $\beta$.

Le fibrinogène est présent chez toutes les espèces animales connues, de la lamproie au primate, et dans tous les cas sa molécule est constituée de trois chaînes polypeptidiques. Cette structure est donc apparue il y a plus de 450 millions d'années. L'analyse des homologies de $m / s n^{\circ} 5$, vol. 2, mai 86 séquence en acides aminés des trois chaînes polypeptidiques indique, en fait, que cette triplication est distante de plus d'un milliard d'années $[18,19]$, approximativement au mo"ment de la séparation entre le végétal et l'animal.

Tout ceci fait du fibrinogène la plus vieille protéine multicaténaire connue et l'organisation de ses gènes pourrait être le résultat de la duplication d'un très vieux gène ancestral, suivi de la transposition et d'une inversion du gène $\beta$.

\section{Fonctions}

Le fibrinogène remplit deux fonctions dans l'hémostase.

Il est d'une part le précurseur de la fibrine dans le système de la coagulation. Il participe, d'autre part, à l'agrégation plaquettaire par l'intermédiaire de récepteurs membra-

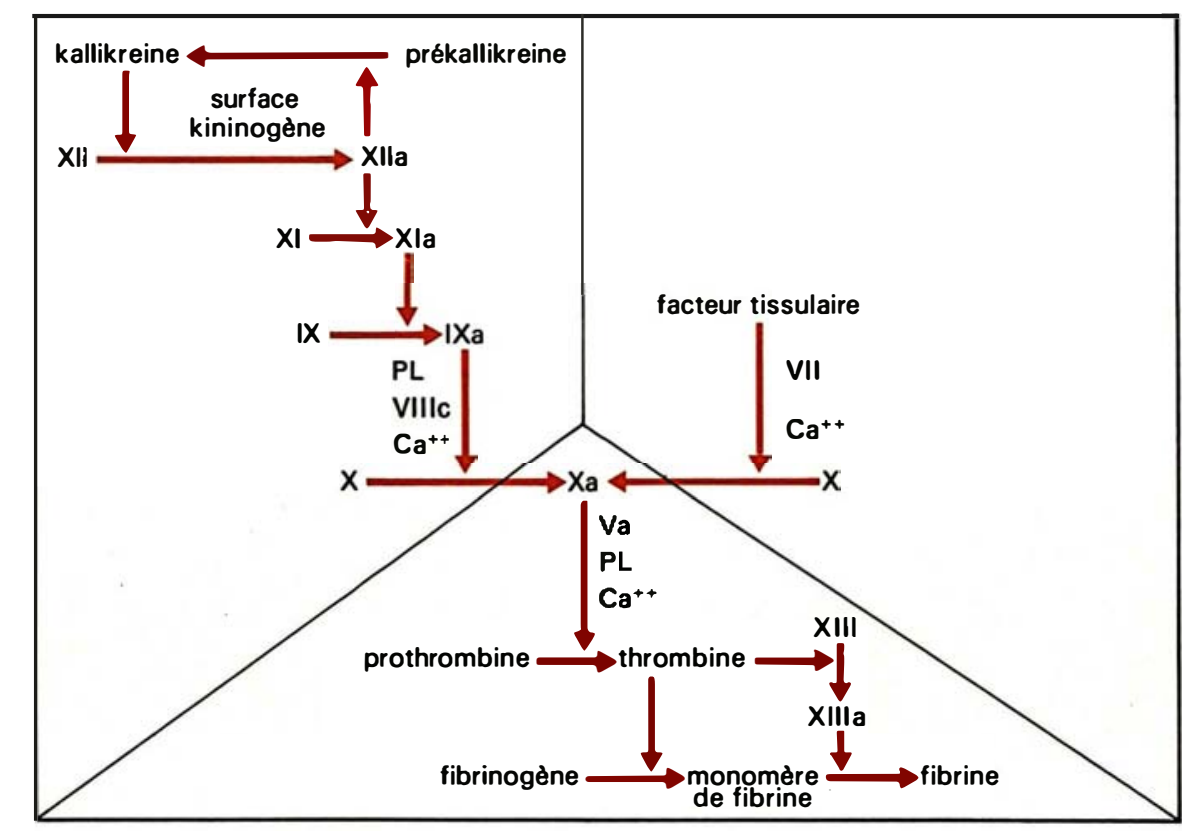

Figure 4. Schéma de la coagulation. Deux mécanismes indépendants, endogène et tissulaire, aboutissent à l'activation protéolytique du facteur $X$ en facteur $X a$. Le facteur $X a$ transforme la prothrombine en thrombine qui agit à son tour sur le fibrinogène pour former le caillot de fibrine. 


\section{REFERENCES}

16. Crabtree GE, Comean CM, Fowlkes DM, Formace AJ, Malley JD, Kant JA. Evolution and structure of fibrinogen genes. $7 \mathrm{Mol} \mathrm{Biol} \mathrm{1985;}$ 185: I-19.

17. Fowlkes D, Mullis NT, Coman CM, Crabtree GR. Potential basis for regulation of the coordinately expressed fibrinogen genes: Homology in the $5^{\prime}$ flanking regions. Proc Natl Acad Sci $U S A$ 1984: 81; 2313-6.

18. Watt KWK, Takagi T, Doolittle RF. Aminoacid sequence of the $\beta$ chain of human fibrinogen. Homology with the $\gamma$ chain. Proc Natl Acad Sci USA 1978; 75: 1731-5.

19. Henshen A, Lottspeich F, Kehl M, Southan $C$, Lucas J. Structure function evolution relationship in fibrinogen. In: Henshen A, de Gruyter W, eds. Fibrinogen-Recent Biochemical and Medical Aspects. Berlin: 1982: 67-82.

20. Marguerie G, Plow EF, Edgington TS. Human platelet possess an inducible and saturable receptor for fibrinogen. $7 \mathrm{Biol}$ Chem 1979; 254: $5357-63$.

21. Marguerie G, Thomas-Maison N, Ginsberg MH, Plow EF. The platelet fibrinogen interaction: evidence for the proximity of the $A \alpha$ chain of fibrinogen to platelet membrane glycoprotein GPIIb-IIIa. Eur 7 Bioch 1984; 139: 5-11.

22. Marguerie G, Plow EF. The fibrinogen dependent pathway of aggregation. Ann NY Acad Sci 1983; 408: 556-66.

23. Nurden AT, Caen JP. An abnormal platelet glycoprotein pattern in three cases of Glanzmann's thrombasthenia. $\mathrm{Br} 7$ Haematol 1974; 38: 233-53.

24. Kloczewiack $M$, Timmons $S$, Lukas $T J$, Hawiger J. Platelet receptor recognition site on human fibrinogen. Biochemistry 1984; 23: 176774

naires hautement spécifiques.

Les changements de structure qui accompagnent la transformation du fibrinogène, protéine soluble, en fibrine insoluble ont été largement étudiés. Cette transformation est le résultat d'une coupure protéolytique des fibrinopeptides A et B par la thrombine. Le monomère de fibrine ainsi formé polymérise pour constituer le caillot de fibrine. Cette fibrine est ensuite stabilisée par des liaisons covalentes grâce à une transglutaminase, le facteur XIIIa ( figure 4); l'identification des sites de polymérisation et de transglutamination est encore l'objet de nombreux travaux car la production d'inhibiteurs de ces sites permettrait de contrôler la formation d'un caillot de fibrine dans de nombreux syndromes thrombotiques.

Le rôle du fibrinogène dans l'agrégation plaquettaire était supposé depuis de nombreuses années, mais le mécanisme de cette activité n'était pas connu. On sait aujourd'hui que la participation du fibrinogène à ce phénomène est due à son interaction avec un récepteur spécifique induit sur la membrane de la plaquette [20] (figure 5). Deux glycoprotéines de la membrane sont impliquées dans cette fonction, la GPIIb et la GPIIIa. Ces deux glycoprotéines forment un complexe stoechiométrique et pourraient constituer le récepteur du fibrinogène. L'utilisation de méthodes de couplage à l'aide de réactifs photosensibles bifonctionnels a permis en effet de montrer que lorsque le fibrinogène est associé à la membrane de la plaquette, il peut être couplé à ce complexe GPIIb-IIIa [2I]. Il est maintenant admis que l'interaction entre le fibrinogène et son récepteur contrôle l'agrégation [22]. Mais le mécanisme exact par lequel la molécule maintient la cohésion des membranes de deux plaquettes adjacentes dans un thrombus n'est pas connu et plusieurs modèles peuvent être proposés ( figure 6). Deux observations attestent de l'importance physiologique de cette agrégation dépendante du fibrinogène : l'induction du récepteur et la fixation du ligand s'observent en milieu plasmatique et pour tous les stimuli physiologiques de la plaquette; cette induction peut impliquer ou ne pas impliquer la réaction de sécrétion qui accompagne la stimulation plaquettaire (figure 5). Par ailleurs, dans la thrombasthénie de Glanzmann, les glycoprotéines IIb et IIIa sont absentes de la membrane plaquettaire [23]; ces plaquettes ne fixent pas le fibrinogène et ne s'agrègent pas en réponse à tous les agonistes connus de la cellule.

Un effort particulier est aujourd'hui consacré à l'identification des structures de la molécule de fibrinogène qui contiennent les sites d'interaction. Ces structures ont été localisées récemment dans les domaines C-terminaux de la protéine. Deux séquences peptidiques, porteuses de l'activité ont été identifiées [24-26]. L'une est localisée dans l'extrémité C-terminale de la chaîne: $\gamma$, l'autre se trouve dans la chaîne $\alpha$. Les recherches en ce domaine se poursuivent, car la production de peptides synthétiques analogues de ces séquences pourrait constituer une voie d'approche pour la mise au point de nouveaux agents thérapeutiques, à exploiter dans les circonstances où l'agrégation plaquettaire devient critique : accident thrombotique, ou développement d'une athérosclérose, ou croissance de tumeur maligne.

Le fibrinogène semblè également jouer un rôle dans les fonctions de la cellule endothéliale. Les cellules endothéliales forment une monocouche non-thrombogénique tout le long du système vasculaire. Elles sont différenciées pour' assurer des échanges rapides entre le flux sanguin et le liquide interstitiel. D'autre part, la migration et la prolifération de cette cellule sont d'une importance majeure pour l'angiogénèse ou la vascularisation des croissances tumorales. Ces phénomènes sont contrôlés par des interactions adhésives entre la membrane de la cellule endothéliale et la matrice du sous-endothélium ou certains facteurs plasmatiques. Nous venons de mettre en évidence une fonction inattendue du fibrinogène. Cette protéine influence de manière spécifique la migration et l'adhérence de la cellule endothéliale [27]. Cette fonction s'exerce par l'intermédiaire de récepteurs dont les" propriétés structurales et immunologiques sont 
comparables à celles des glycoprotéines plaquettaires IIb et IIIa. Il semblerait donc que le récepteur du fibrinogène soit synthétisé dans des systèmes cellulaires autres que le mégacaryocyte. La réalité physiologique d'une telle fonction et la structure de ces récepteurs sont en cours d'étude.

\section{Physiopathologie moléculaire}

Il existe trois anomalies congénitales associées à la molécule de fibrinogène. Les afibrinogénémies se caractérisent par une absence totale de fibrinogène plasmatique et plaquettaire. Elles sont causes d'hémorragies graves qui surviennent dès la naissance. Il s'agit d'une maladie rare, mais non exceptionnelle, bien caractérisée du point de vue clinique et biologique, et dont le mode de transmission est autosomal récessif. Les mécanismes qui sont à l'origine des afibrinogénémies sont inconnus. Dans notre laboratoire nous avons analysé l'ADN de quelques afibrinogénémiques. Les résultats obtenus sur huit malades ont montré que les gènes du fibrinogène sont présents et aucune délétion génique ou remaniement important n'a pu être mis en évidence. Ceci indique que chez ces patients la maladie est causée par un défaut plus subtil, minidélétion,

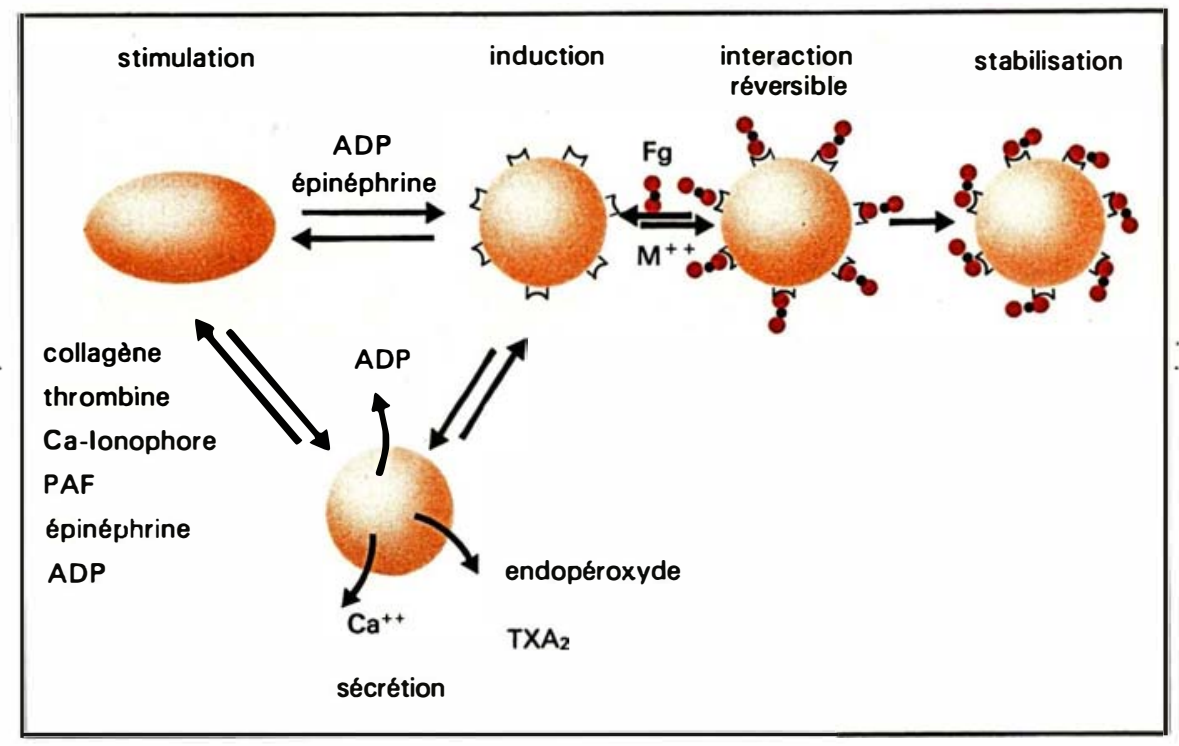

Figure 5. Schéma réactionnel de l'interaction du fibrinogène avec son récepteur plaquettaire. Cette interaction comporte quatre étapes: 1) stimulation de la plaquette circulante, 2) induction du récepteur selon une voie non dépendante de la sécrétion et une voie dépendante de la production d'endopéroxyde et de la sécrétion d'ADP et de calcium intracellulaire, 3) interaction réversible du fibrinogène avec son récepteur et 4) stabilisation $d u$ complexe. PAF= platelet activating factor.

mutation ponctuelle ou défaut d'origine pré ou post-traductionnelle.

Les hypofibrinogénémies, de transmission autosomale dominante, se traduisent par une diminution du taux de fibrinogène plasmatique. Deux mécanismes peuvent être à l'origine de cette déficience quanti- tative. Elle peut être due à un défaut de sécrétion avec accumulation de la protéine synthétisée dans l'hépatocyte. Elle peut être également due à une sensibilité particulière de la protéine anormale à la protéolyse. Très souvent les hypofibrinogénémies sont associées aux

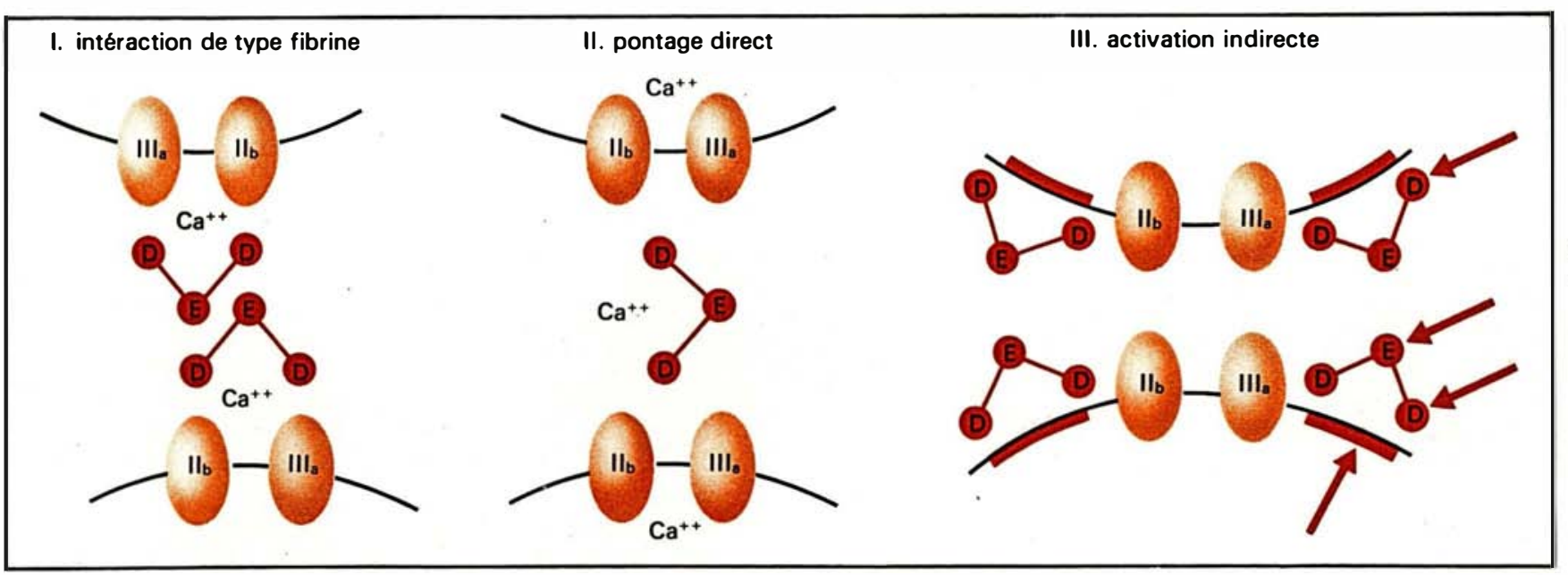

Figure 6. Mécanismes hypothétiques par lesquels le fibrinogène et son récepteur contrôlent l'agrégation plaquettaire. I et /I représentent deux modèles qui impliquent une interaction directe du ligand avec le complexe GPIIb-IIla et un pontage entre deux membranes cellulaires adjacentes, III représente une interaction indirecte et une activation du complexe GPIIb-IIla qui maintiendrait la cohésion de deux membranes. 
dysfibrinogénémies.

Les dysfibrinogénémies sont liées à une anomalie fonctionnelle dont les causes sont multiples : mutation ponctuelle affectant un acide aminé fonctionnellement important; élongation ou réduction des chaînes $\beta$ ou $\gamma$. Ces anomalies correspondent à des mutations, des délétions ou des défauts de l'expression de la protéine dont les mécanismes sont encore à découvrir.

L'un des aspects importants de l'analyse clinique du fibrinogène concerne également l'identification et la quantification de certains dérivés physiologiques de cette protéine. Dans certaines affections acquises, il peut $\mathrm{y}$ avoir activation de la coagulation in vivo, génération d'une faible quantité de thrombine et production de monomères de fibrine. Ces monomères ont une tendance à polymériser et s'échappent dans la circulation générale sous forme de complexes solubles. La détection de ces complexes est utile pour le diagnostic d'une coagulation intravasculaire disséminée, bien qu'elle ne permette pas d'établir un diagnostic causal. Il existe très peu de méthodes fiables et sensibles pour cette mesure. Le développement de dosages immunochimiques spécifiques des peptides $\mathrm{A}$ et $B$ permet d'évaluer par des tests Elisa l'action d'une thrombine active. Mais des doutes persistent quant à la spécificité de ces tests qui sont plus avantageusement utilisés pour suivre les effets d'une thérapeutique anticoagulante. Par ailleurs, l'action de la plasmine génère des produits de dégradation du fibrinogène ou de la fibrine qui peuvent donc circuler en même temps que ces complexes solubles. Il est donc difficile de distinguer de manière spécifique une action fibrinolytique d'une action thrombotique. La tendance actuelle est d'essayer de développer des anticorps monoclonaux qui reconnaissent des produits de dégradation spécifiques du fibrinogène ou de la fibrine. De tels anticorps ont déjà été caractérisés [28]. Ils semblent constituer un bon outil pour une meilleure analyse des syndromes associés à des coagulations intravasculaires ou à des fibrinolyses normales ou induites

\section{Summary}

Fibrinogen is a major component of the blood coagulation system. The plasma constitutes the major source of the protein which is also present and stored in platelet $\alpha$ granules. The molecule is the product of three separate genes, located on chromosome 4 , coding for the three constitutive polypeptidic chains. The plasma molecule is synthesized and secreted by the hepatocyte, whereas the platelet fibrinogen is produced in the megakaryocyte. Differences between the two molecules hrive been reported suggesting that expression of fibrinogen in the two cellular compartments may be different. Over the pasi thirty years, a large body of information on fibrinogen - has been accumulated and has shown this protein to be a multifunctional molecule. The reactions with thrombin, plasmin, and factor XIIIa have been fully characterized. The role of the protein in platelet aggregation and endothelial cell migretion and proliferation via specific membrane associated receptors have been identified. The bulk of information now available on this protein has greatly:motivated new investigations : on the physiology and the pathophysiology of this factor at the cellular level.

\section{TIRES A PART}

G. Marguerie : Département de reclierche fondamentale, laboratoire d'hématologie, unité Inserm 217, C.E.N.G.-85 X, 38041 Grenoble Cedex.

$$
m / s n^{\circ} 5, \text { vol. 2, mai } 86
$$

\section{gen, Structure, Functional Aspects, Metabolism. 1983; 2: 227-33 \\ 28. Soria J, et al. Immunochemical differen- tiation of fibrinogen fragments $\mathrm{D}$ and $\mathrm{E}$ and cross} (2) 